\title{
Live-Cell Imaging and Quantification of PolyQ Aggregates by Stimulated Raman Scattering of Selective Deuterium Labeling
}

\author{
Kun Miao and Lu Wei*
}

Cite This: ACS Cent. Sci. 2020, 6, 478-486

Read Online

ABSTRACT: Polyglutamine (polyQ) diseases are a group of neurodegenerative disorders, involving the deposition of aggregation-prone proteins with long polyQ expansions. However, the cytotoxic roles of these aggregates remain highly controversial, largely due to a lack of proper tools for quantitative and nonperturbative interrogations. Common methods including in vitro biochemical, spectroscopic assays, and live-cell fluorescence imaging all suffer from certain limitations. Here, we propose coupling stimulated Raman scattering microscopy with deuteriumlabeled glutamine for live-cell imaging, quantification, and spectral analysis of native polyQ aggregates with subcellular resolution.

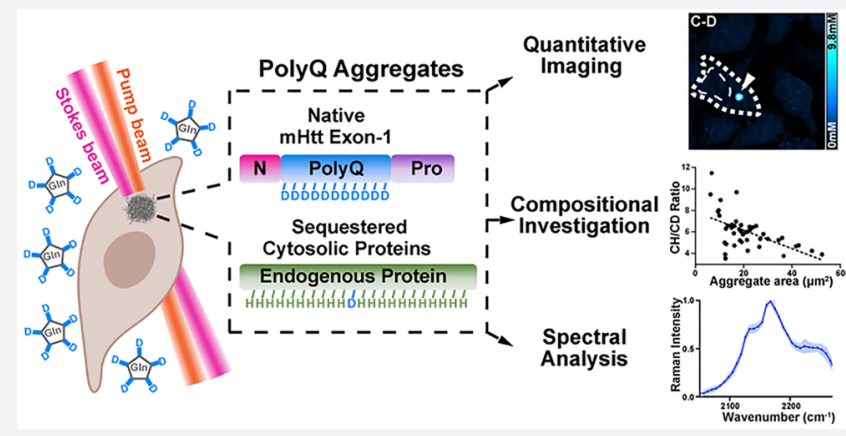
First, through the enrichment of deuterated glutamine in the polyQ sequence of mutant Huntingtin ( $\mathrm{mHtt}$ ) exon1 proteins for Huntington's disease, we achieved sensitive and specific stimulated Raman scattering (SRS) imaging of carbon-deuterium bonds (C-D) from aggregates without GFP labeling, which is commonly employed in fluorescence microscopy. We revealed that these aggregates became 1.8-fold denser compared to those with GFP. Second, we performed ratiometric quantifications, which indicate a surprising dependence of protein compositions on aggregation sizes. Our further calculations, for the first time, reported the absolute concentrations for sequestered $\mathrm{mHtt}$ and non-mHtt proteins within the same aggregates. Third, we adopted hyperspectral SRS for Raman spectroscopic studies of aggregate structures. By inducing a cellular heat shock response, a potential therapeutic approach for inhibiting aggregate formation, we found a possible aggregate intermediate state with changed solvation microenvironments. Our method may hence readily unveil new features and mechanistic insight of polyQ aggregates and pave the way for comprehensive in vivo investigations.

\section{INTRODUCTION}

A hallmark of neurodegenerative disorders is the presence of protein aggregates in peripheral nerves. ${ }^{1-4}$ Among these disorders are polyglutamine (polyQ) diseases, such as Huntington's disease (HD), which starts with motor symptoms like chorea and is followed by memory deficit and depression. ${ }^{3,4}$ The onset of HD has been linked to abnormally expanded CAG trinucleotide repeats that encode the polyQ sequence in mutant Huntingtin ( $\mathrm{mHtt}$ ) proteins. While $\mathrm{Q}$ repeats are typically fewer than 37 in healthy humans, they can range from 40 to 250 in Huntington's patients and are consistently found in the protein depositions of HD brain slices by immunohistology. ${ }^{1,3}$ However, the pathological roles of polyQ aggregates still remain elusive., ${ }^{2,-6}$ Recent studies suggest that soluble oligomers are cytotoxic by dynamically interacting with cytosolic proteins and triggering apoptosis while aggregates are cytoprotective by sequestering toxic protein oligomers to form stable inclusion bodies. ${ }^{4,7,8}$ In contrast, evidence also indicates that toxicity of aggregates arises from depleting functional (e.g., chaperones and transcription factors) and structural (e.g., actin) proteins and impairing cellular organelles (e.g., ribosomes and endoplasmic reticulum). ${ }^{9-13}$
To understand their molecular roles, extensive efforts have been made to investigate the compositions, structures, and kinetics of $\mathrm{mHtt}$ aggregates. Conventional biochemical assays and recent quantitative proteomics offer relative protein compositions of the aggregates in reference to the soluble protein pools. However, these methods rely on extensive postprocessing such as aggregation purification and solubilization. ${ }^{9,10}$ In vitro spectroscopic studies including IR, ${ }^{14} \mathrm{UV}$ resonance Raman, ${ }^{15,16}$ NMR spectroscopy, ${ }^{17}$ and fluorescence $^{18,19}$ on model peptides provide crucial information, but they are limited to relatively short expansion lengths because of the difficulty in isolating peptides with long $Q$ repeats. ${ }^{14-19}$ More importantly, all these in vitro studies cannot recapitulate the native aggregation status in live cells. For live-cell studies, fluorescence imaging offers unprecedented spatial and

Received: November 19, 2019

Published: March 6, 2020 

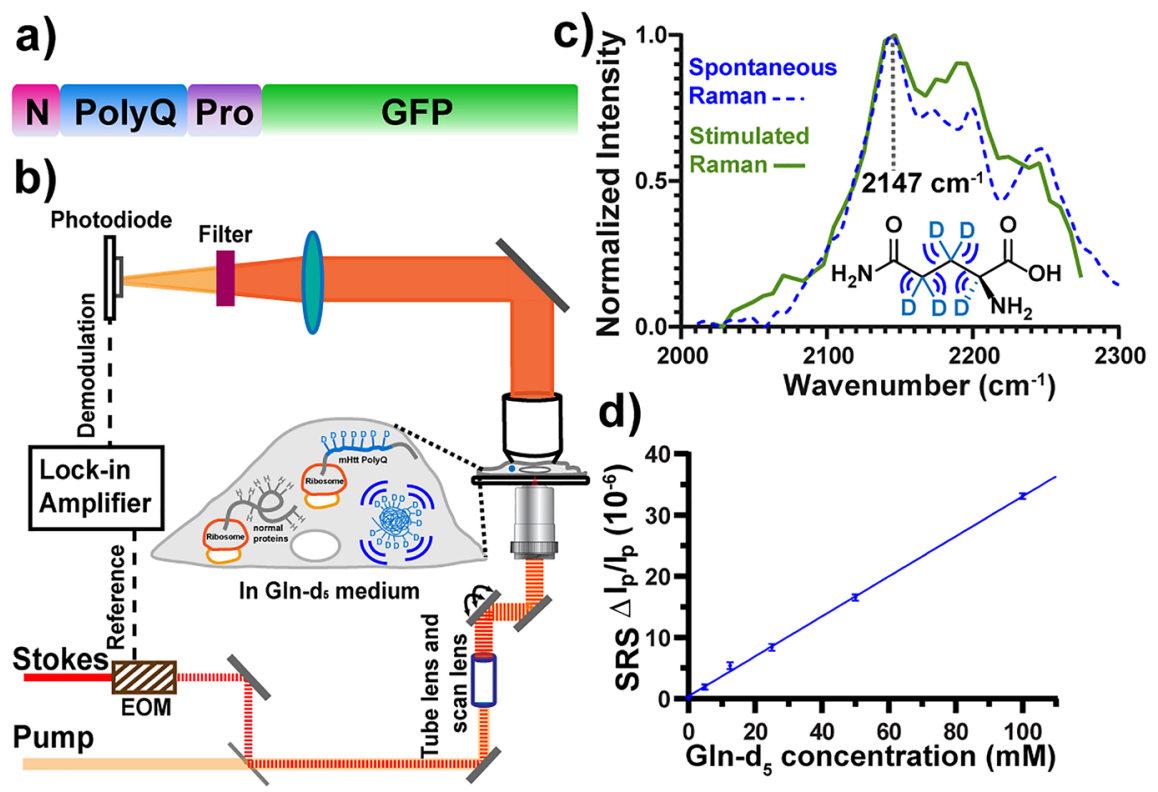

Figure 1. Experimental scheme for stimulated Raman scattering (SRS) microscopy with deuterated glutamine (Gln or Q) labeling. (a) Plasmid construct of a model mutant Huntingtin ( $\mathrm{mHtt}$ ) Exon1 (ex1) protein fused with GFP at the $\mathrm{C}$ terminus. N: N-terminal 17 aa fragment. PolyQ: poly glutamine region. Pro: proline-rich domain. GFP: green fluorescent protein. (b) Experimental scheme for SRS imaging of Gln- $d_{5}$-labeled polyQ aggregates. (c) Spontaneous Raman (blue dashed) and SRS (green) spectra of $60 \mathrm{mM}$ Gln- $d_{5}$ solution. (d) Linear dependence of SRS signals (at $2147 \mathrm{~cm}^{-1}$ ) on Gln- $d_{5}$ concentrations under a $50 \mu$ s time constant. Error bar: SD.

temporal resolution, by fusing fluorescent proteins ${ }^{20}$ or selflabeling tags (e.g., HaloTag) ${ }^{2 \mathrm{O}}$ to the C-terminus of a $\mathrm{mHtt}$ exon1 (ex1) sequence (Figure 1a). The aggregation-prone ex1 fragment, which comprises a $17 \mathrm{~N}$-terminal sequence, a polyQ tract followed by a proline-rich domain at the C-terminus (Figure 1a), can effectively induce the pathological phenotype of $\mathrm{HD}$ in the transgenic mouse model and humans. ${ }^{4,22}$ Compared to $\mathrm{mHtt}$ exl, however, green fluorescent protein (GFP) is much larger in size and has a known tendency to oligomerize. ${ }^{23}$ This could perturb the aggregation kinetics and conformations and may contribute to the controversy of reported toxicity. Moreover, because the dense aggregation environment often causes fluorescence quenching, ${ }^{24}$ fluorescence imaging is not ideal for quantitative analysis of aggregates. It is therefore highly desirable to have a new modality that combines the advantages from in vitro investigations and fluorescence imaging while overcoming their limitations.

Here, we report a novel and general platform for live-cell imaging, quantification, and spectral analysis of polyQ aggregates by stimulated Raman scattering (SRS) microscopy (Figure $\mathrm{S} 1$ ) of deuterium-labeled glutamine $\left(\mathrm{Gln}-d_{5}\right)$ (Figure $1 b, c)$. We first achieved specific and sensitive SRS imaging of native polyQ aggregates without the need of large fusionproteins. We then developed a ratiometric strategy for quantitative analysis of protein content in aggregates of varying sizes. Compared to existing methods, our modality, for the first time, reports the absolute concentrations of both $\mathrm{mHtt}$ and non-mHtt proteins from aggregates in live cells. Applying the hyperspectral SRS (hSRS), we further probed the aggregate structures and aggregate-environment interactions upon an induced heat shock response.

\section{RESULTS}

Coupling of SRS Microscopy with Deuterated Glutamine Is Ideal for Imaging PolyQ Aggregates.
Labeling of aggregates is achieved through replacing regular Gln in the medium with Gln- $d_{5}$ (Figure $1 \mathrm{~b}, \mathrm{c}$ ), which would be metabolically incorporated and enriched into the long polyQ tail of expressed mHtt proteins (Figure la,b). Targeting the vibrational frequency of carbon-deuterium bonds (C-D), SRS imaging obtains subcellular mapping of $\mathrm{mHtt}$ aggregates in live cells. Our strategy has the following advantages: First, the vibrational frequency of $\mathrm{C}-\mathrm{D}$ in Gln- $d_{5}$ (Figure 1c) is in the desired cell-silent region $\left(1800-2600 \mathrm{~cm}^{-1}\right)$, providing high imaging specificity without background from endogenous biomolecules. Second, the nonexchangeable labeling on the $\mathrm{C}-\mathrm{H}$ side chains of Gln offers reliable signals. Third, Gln enrichment in the polyQ region (Figures 1a) yields both high labeling specificity and superb SRS imaging sensitivity. For example, for a widely used mHtt-97Q exl protein, Gln accounts for $68 \%$ of the ex1 sequence (Figure S2), while the natural occurrence of Gln is only $4.2 \%$ in human proteomes. ${ }^{25}$ Compared to label-free $\mathrm{SRS}^{26}$ and SRS imaging with ${ }^{13} \mathrm{C}$ phenyalanine $^{27}$ or deuterated all essential amino acids, ${ }^{28}$ selective Gln- $d_{5}$ labeling is significantly more specific for imaging polyQ aggregates. Fourth, compared to using alkynetagged unnatural amino acids, which only introduces one tag to one copy of a protein, ${ }^{29}$ multiple Q labeling (e.g., $103 \mathrm{Q}$ for mHtt-97Q ex1) has higher sensitivity and requires less sample manipulation. Fifth, imaging-wise, compared to spontaneous Raman, ${ }^{30}$ SRS provides higher detection sensitivity and faster image acquisition with the stimulated emission quantum amplification principle (Figure S1). Compared to Coherent anti-Stokes Raman scattering (CARS), ${ }^{31}$ another nonlinear Raman microscopy technique, SRS, offers high-fidelity Raman spectra (Figure 1c) and linear concentration dependence (Figure 1d) without a nonresonance background.

We first determined our SRS detection limit on Gln- $d_{5}$ solution to be $3 \mathrm{mM}$ (Figure $1 \mathrm{~d}$, when signal $(S) /$ noise $(N)=$ 1) by targeting the $\mathrm{C}-\mathrm{D}$ peak at $2147 \mathrm{~cm}^{-1}$. Hence, our detection limit is as low as $29 \mu \mathrm{M}$ for $\mathrm{mHtt}$ proteins with a 


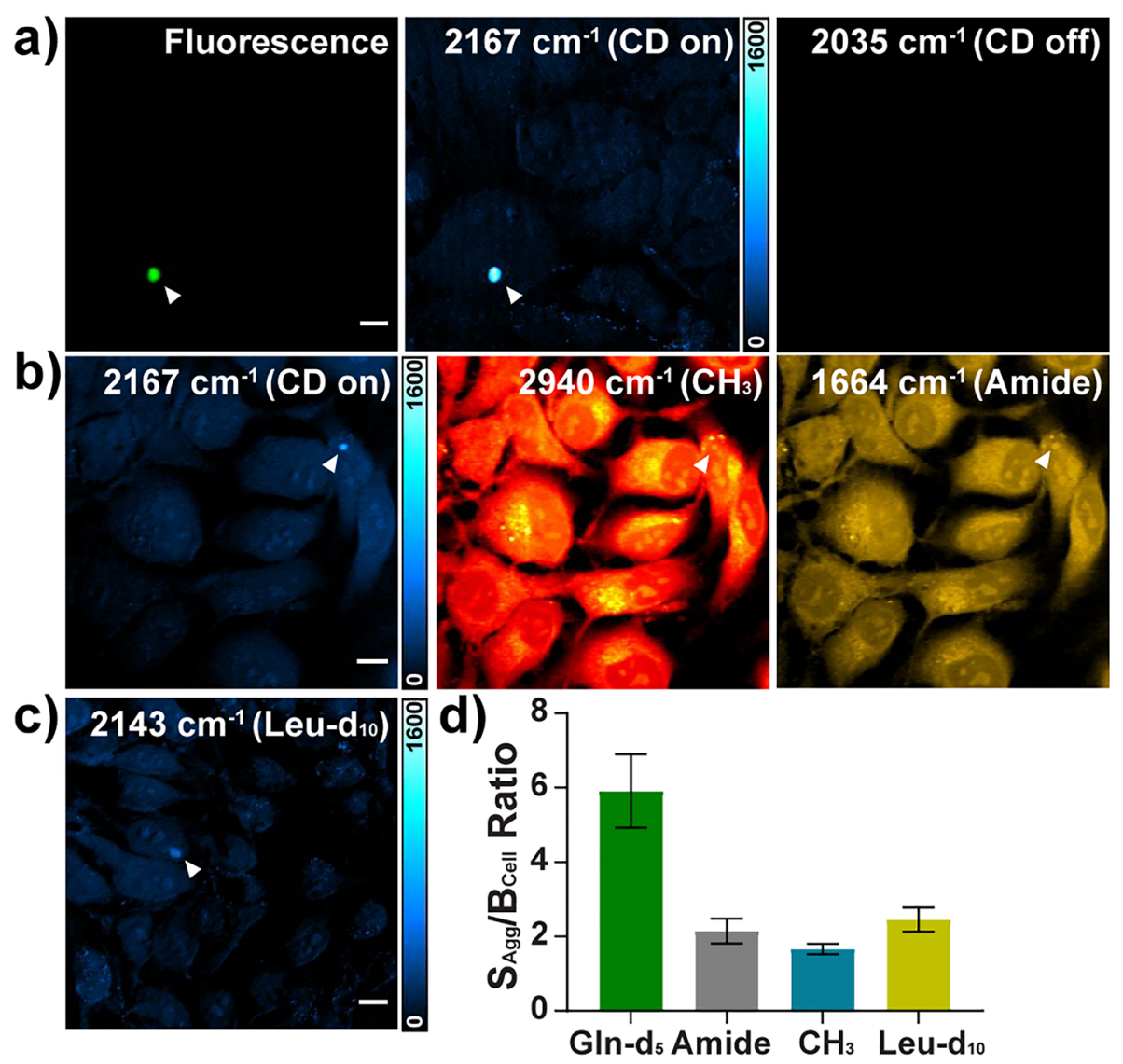

Figure 2. Live-cell SRS imaging of mHtt-97Q-GFP aggregates with Gln- $d_{5}$ labeling. (a) SRS imaging of mHtt aggregates (arrowheaded, $2167 \mathrm{~cm}{ }^{-1}$, $\mathrm{C}-\mathrm{D}$ on), validated by fluorescence imaging through GFP (Fluorescence). Off-resonance image at $2035 \mathrm{~cm}^{-1}$ shows no signal. (b) Live-cell SRS images for an mHtt-97Q-GFP aggregate (arrowheaded) at Gln- $d_{5}\left(2167 \mathrm{~cm}^{-1}\right), \mathrm{CH}_{3}\left(2940 \mathrm{~cm}^{-1}\right)$, and amide I $\left(1664 \mathrm{~cm}^{-1}\right) \mathrm{channels}$ on the same set of HeLa cells. (c) SRS imaging of an mHtt-97Q-GFP aggregate at $2143 \mathrm{~cm}^{-1}$ by leucine- $d_{10}$ (Leu- $d_{10}$ ) labeling. (d) Average $S_{\text {agg }} / B_{\text {cell }}$ from SRS images of C-D with Gln- $d_{5}$ labeling $(5.75 \pm 1.03, n=13)$; amide I $(2.15 \pm 0.34, n=4) ; \mathrm{CH}_{3}(1.66 \pm 0.14, n=10)$; and C-D with Leu- $d_{10}$ labeling $(2.45 \pm 0.33, n=10)$. Error bar: SD.

total of $103 \mathrm{Gln}$ in the $\mathrm{mHtt}-97 \mathrm{Q}$ ex 1 protein. This strategy offers higher detection sensitivity compared to previously reported $200 \mu \mathrm{M}$ by SRS imaging of alkyne tags. ${ }^{32}$ In cells, however, the detection limit is set by aggregate-signal to cellular-background ratio $\left(S_{\text {agg }} / B_{\text {cell }}\right)$. There are two sources for $B_{\text {cell }}$. First, the newly synthesized proteome incorporates Gln. Since Gln accounts for $4.2 \%$ of human proteome, ${ }^{25}$ the estimated detectability for mHtt-97Q ex1 is $86 \mu \mathrm{M}$ when $S_{\text {agg }} /$ $B_{\text {cell }}=1$ (details in the SI). Second, the intracellular free Gln pool is about $8 \mathrm{mM}^{33}$ slightly lowering the achievable detectability. To minimize this additional background, we replaced the medium with buffer before imaging. Although a detection sensitivity of $86 \mu \mathrm{M}$ may be too high for imaging normal proteins, it is highly feasible to detect polyQ aggregates down to very small sizes. Our GFP fluorescence analysis by time-lapse tracking of mHtt-97Q-GFP proteins (Figure S3a,b) and tetramethylrhodamine (TMR) fluorescence analysis of $\mathrm{mHtt}-97 \mathrm{Q}-\mathrm{Hal}$ proteins (Figure S3c) suggest that once the new and small aggregates form in cells, the concentration of polyQ protein is already beyond $86 \mu \mathrm{M}$ to as high as $400 \mu \mathrm{M}$.

Imaging and Quantification of $\mathrm{mHtt}$ Aggregates with and without GFP Labeling in Live HeLa Cells. We next validated our SRS imaging by transfecting HeLa cells with mHtt-97Q-GFP plasmid (Figure $1 \mathrm{a}$ and Figure S2) and culturing them in Gln- $d_{5}$ medium. We first conducted parallel SRS and fluorescence imaging on the same set of live cells. The
SRS image of C-D enriched aggregate (Figure 2a, $2167 \mathrm{~cm}^{-1}$ ) agrees well with the fluorescence image of GFP (Figure 2a, fluorescence). A clear off-resonance image demonstrates high SRS imaging quality (Figure 2a, $2035 \mathrm{~cm}^{-1}$ ). The $\mathrm{C}-\mathrm{D}$ peak for Gln- $d_{5}$ is shifted from $2147 \mathrm{~cm}^{-1}$ in solution to $2167 \mathrm{~cm}^{-1}$ after being incorporated into cellular proteins, suggesting a change of the microenvironment. Indeed, we found that the shifted C-D spectrum has Raman spectral features from both Gln- $d_{5}$ solution and solid (peaked at $2167 \mathrm{~cm}^{-1}$ ) (Figure S4). As a comparison, label-free SRS images at $\mathrm{CH}_{3}\left(2940 \mathrm{~cm}^{-1}\right)$ and amide I $\left(1664 \mathrm{~cm}^{-1}\right)$ channels, widely adopted for imaging total proteins, ${ }^{26}$ show much decreased detection specificity, particularly pronounced for small aggregates (Figure $2 b$, arrow indicated), which are indistinguishable from the nucleoli. Moreover, the high contrast of the $\mathrm{C}-\mathrm{D}$ image decreases significantly if Gln- $d_{5}$ is replaced by nonenriched leucine- $d_{10}$ (Leu- $d_{10}$ ) for labeling (Figure 2c). Our quantification on the average $S_{\text {agg }} / B_{\text {cell }}$ for each channel clearly demonstrated much higher imaging specificity with Gln- $d_{5}$ (Figure $2 \mathrm{~d}$ ).

After establishing the feasibility for SRS imaging of Gln- $d_{5}$ labeled mHtt-97Q-GFP aggregates, we aimed to image native mHtt-97Q proteins without GFP (Figure 3a). GFP may perturb aggregation formation of $\mathrm{mHtt}$ proteins, because it is 238 amino acid (aa) in length, which is about twice as large as mHtt-97Q ex1 with only 152 aa. We successfully imaged aggregates at the same $\mathrm{C}-\mathrm{D}$ frequency (Figure $3 \mathrm{~b}, \mathrm{CD}$ on and 


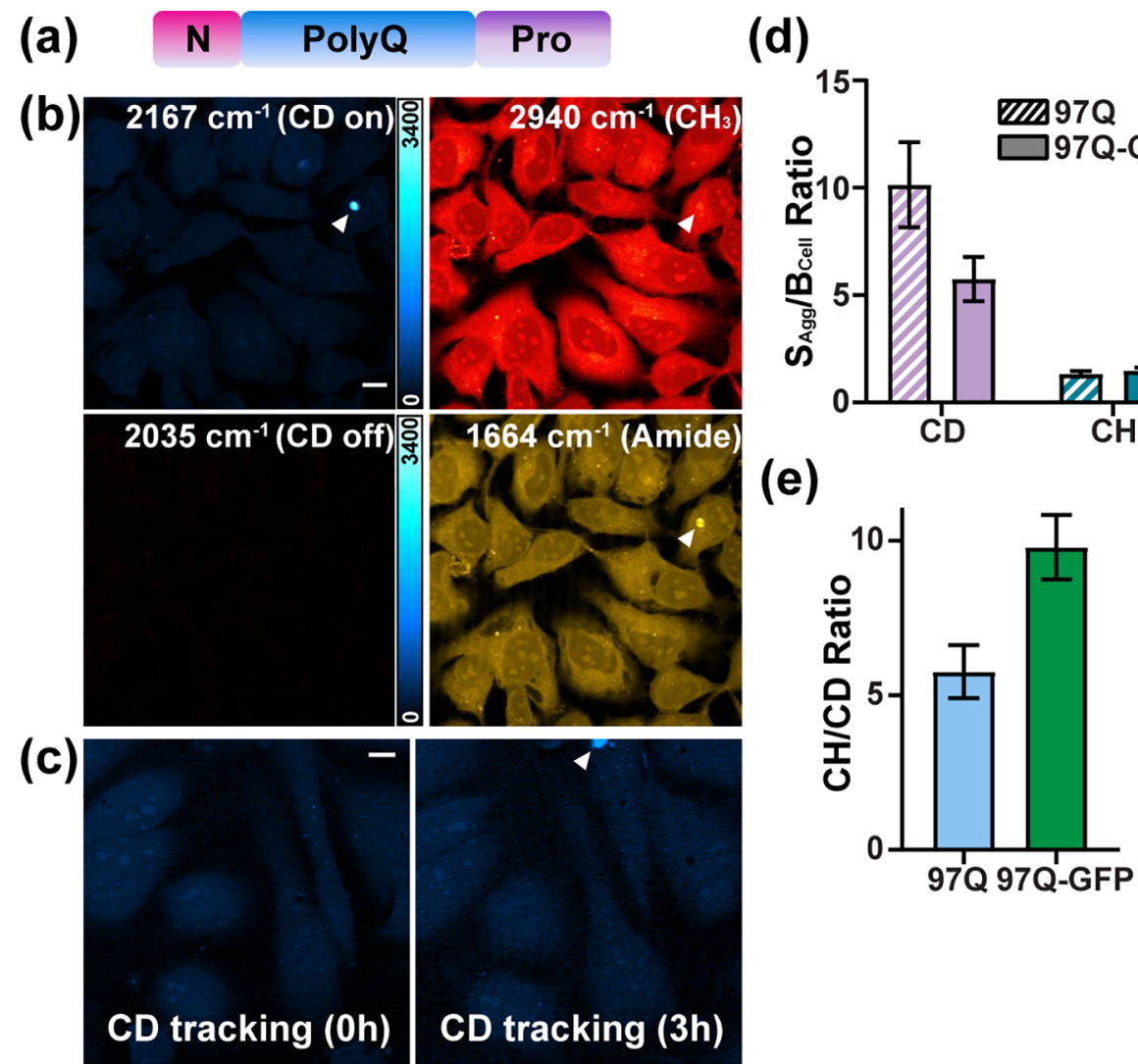

Figure 3. SRS imaging of $\mathrm{mHtt}-97 \mathrm{Q}$ aggregates without GFP. (a) Plasmid construct of mHtt-97Q by deleting GFP sequence. (b) SRS images of a Gln- $d_{5}$-labeled aggregate at C-D on-resonance $\left(2167 \mathrm{~cm}^{-1}\right), \mathrm{C}-\mathrm{D}$ off-resonance $\left(2035 \mathrm{~cm}^{-1}\right), \mathrm{CH}_{3}\left(2940 \mathrm{~cm}^{-1}\right)$, and amide I $\left(1664 \mathrm{~cm}^{-1}\right)$. $(\mathrm{c})$ Time-lapse SRS tracking at the C-D channel for capturing aggregation formation on the same set of live cells. (d) $S_{\text {agg }} / B_{\text {cell }}$ of $\mathrm{mHtt}-97 \mathrm{Q}(97 \mathrm{Q})$ in $\mathrm{C}-\mathrm{D}(\mathrm{CD}, 10.14 \pm 1.99, n=11)$ and $\mathrm{CH}_{3}(\mathrm{CH}, 1.50 \pm 0.12, n=10)$ channels compared to that for mHtt-97Q-GFP $(97 \mathrm{Q}-\mathrm{GFP}, \mathrm{CD}, 5.75 \pm 1.03$, $n=13$; CH, $1.66 \pm 0.14, n=10)$. (e) CH/CDs for 97Q-GFP $(9.79 \pm 1.05, n=7)$ and 97Q $(5.75 \pm 0.86, n=20)$ aggregates. Scale bar: $10 \mu \mathrm{m}$. Error bar: SD.

off). Interestingly, these aggregates (Figure $3 \mathrm{~b}, 2167 \mathrm{~cm}^{-1}$ ) are about twice as bright as those of similar sizes with GFP labeling (Figure 2a, $2167 \mathrm{~cm}^{-1}$ ). Because the GFP sequence only contains $8 \mathrm{Gln}$, the $\mathrm{C}-\mathrm{D}$ intensity should remain approximately unchanged when deleting GFP. We hence reasoned that the detected intensity increase is due to the formation of denser aggregates. A similar phenomenon was recently reported by Cryo-ET with the same mHtt exl sequence. ${ }^{11}$ To test our theory, we acquired amide I images to compare total protein concentrations between aggregates with and without GFP. If the density remains unchanged, $\mathrm{mHtt}-97 \mathrm{Q}$ aggregates would have a much lower amide intensity than mHtt-97Q-GFP because the GFP sequence contributes significantly to amide signals. We observed similar levels of amide signals between these two types of aggregates (Figure S5), confirming that the increase in aggregate density makes up for the signal loss of deleting GFP. Moreover, mHtt-97Q aggregates become barely distinguishable from cellular background in the $\mathrm{CH}_{3}$ channel (Figure 3b, $2940 \mathrm{~cm}^{-1}$ ), indicating that previous $\mathrm{CH}_{3}$ signals for aggregates were mainly from the GFP sequence (Figure $2 \mathrm{~b}, 2940 \mathrm{~cm}^{-1}$, and Figure S2). To prove the high biocompatibility of our method, we also demonstrated time-lapse SRS imaging on the same set of live cells to capture the aggregation formation (Figure 3c). To quantify aggregates from multiple experimental replicates, we plotted the average $S_{\text {agg }} / B_{\text {cell }}$ in both $\mathrm{C}-\mathrm{D}$ and $\mathrm{CH}$ channels for $\mathrm{mHtt}-97 \mathrm{Q}$ and $\mathrm{mHtt}-97 \mathrm{Q}-\mathrm{GFP}$ aggregates (Figure $3 \mathrm{~d}$ ). In the C-D channel, the $S_{\text {agg }} / B_{\text {cell }}$ for $\mathrm{mHtt}-97 \mathrm{Q}$ aggregates is 1.8 -fold higher, confirming the formation of 1.8 -fold denser aggregates without GFP labeling, as $B_{\text {cell }}$ is not affected by the change of plasmids. In the $\mathrm{C}-\mathrm{H}$ channel, the $S_{\text {agg }} / B_{\text {cell }}$ values remain approximately the same. We further confirmed our high detection specificity of polyQ aggregates by inducing the formation of stress granules in normal HeLa cells and confirmed that our SRS imaging with Gln- $d_{5}$ labeling does not detect these non-polyQ-aggregate-type of stress granules (Figure S6). Going beyond mHtt-97Q we imaged and quantitatively analyzed a shorter $\mathrm{Q}$ expansion sequence, mHtt-46Q for which the $Q$ number is just above the disease-prone aggregation threshold (Figure S7). Similarly, we found that $\mathrm{mHtt}-46 \mathrm{Q}$ aggregates are about 1.6-time denser compared to those formed by mHtt-46Q-GFP. Moreover, we demonstrated our applicability of imaging polyQ aggregates in a stable embryonic stem cell-line ${ }^{34}$ (Figure S8), paving the way for more general future applications.

Ratiometric Quantifications for the Dependence of Protein Compositions on Aggregation Sizes. The feature of parallel $\mathrm{C}-\mathrm{D}$ and $\mathrm{CH}_{3}$ SRS imaging enables us to perform ratiometric analysis of $\mathrm{CH}_{3}$-to- $\mathrm{CD}$ ratios ( $\mathrm{CH} / \mathrm{CDs}$ ) to investigate polyQ aggregate compositions of non-mHtt and mHtt proteins. This is because the CD signals are from Gln- $d_{5}$, which mainly originates from mHtt proteins, while the $\mathrm{CH}_{3}$ signals represent non-Gln aa, which come from the combined sources of non-Gln aa in mHtt proteins and sequestered non- 
mHtt proteins. We first applied $\mathrm{CH} / \mathrm{CD}$ quantifications on both $\mathrm{mHtt}-97 \mathrm{Q}-\mathrm{GFP}$ and $\mathrm{mHtt}-97 \mathrm{Q}$ aggregates (Figure $3 \mathrm{e}$ ). Surprisingly, the average $\mathrm{CH} / \mathrm{CD}$ remains as high as 5.75 for $\mathrm{mHtt}-97 \mathrm{Q}$ aggregates (Figure $3 \mathrm{e}$ ). If the aggregates were formed primarily by $\mathrm{mHtt}$ proteins, the $\mathrm{CH} / \mathrm{CD}$ should be about 0.5 because the mHtt-97Q sequence contains 49 non- $Q$ aa and 103 Q. Similarly, the $\mathrm{CH} / \mathrm{CD}$ should have decreased by 5-fold from mHtt-97Q-GFP (289 non-Q aa and $112 \mathrm{Q}$ ) to $\mathrm{mHtt}-97 \mathrm{Q}$ aggregates (49 non-Q aa and $103 \mathrm{Q}$ ) (Figure S2). Instead, the decrease is only 1.5 -fold (Figure 3e, 9.79 vs 5.75 ). Our control experiments confirmed that the Gln- $d_{5}$ labeling efficiency is close to $100 \%$ (i.e., de novo glutamine synthesis for regular Gln is about zero, Figures S9 and S10), indicating that all $103 \mathrm{Q}$ in the $\mathrm{mHtt}$ sequence are deuterated. Our results hence imply that these aggregates contain a rather high percentage of non-mHtt proteins. Sequestrations and depletions of cellular functional and structural proteins by aggregates have been suggested to be one underlying mechanism of $\mathrm{HD}$ cytotoxicity. ${ }^{9-13}$ Consequently, we calculated the relative molar percentages for $\mathrm{mHtt}$ and non-mHtt proteins in the aggregates. Assuming that the molar percentage of $\mathrm{mHtt}$ and non-mHtt proteins in the aggregates is $x$ and $1-x$, respectively, with known protein sequences, we can generate an equation with $x$ and $(1-x)$ for the relative ratio between non-Gln aa and Gln- $d_{5}$ to represent the measurable of $\mathrm{CH}$ / CDs (Figure 3e) (details in the SI). We calculated that the average molar percentage of non- $\mathrm{mHtt}$ proteins is indeed as high as $54 \%$ for an average $\mathrm{CH} / \mathrm{CD}$ of 5.75 .

Now that we have established that $\mathrm{CH} / \mathrm{CD}$ s could serve as a direct indicator of aggregation compositions, we next examined the relationship between $\mathrm{CH} / \mathrm{CDs}$ and aggregation sizes. Based on the sequestration theory, ${ }^{12,13}$ we expect that the relationship might offer insight for understanding the molecular cytotoxicity of polyQ aggregates. Interestingly, we observed a negative correlation (Figure 4a, Pearson's $r=$ $-0.56)$, which indicates that the percentage of sequestered $\mathrm{mHtt}$ proteins increases as the aggregates grow. As a comparison, such distinctive correlation by Gln- $d_{5}$ labeling is absent when replaced by Leu- $d_{10}$ labeling (Figure $4 \mathrm{~b}$, Pearson's $r=-0.11$ ), because $\mathrm{mHtt}$ and non-mHtt proteins have similar leucine abundance. This underscores the importance of specific polyQ labeling to observe such a size-composition correlation.

In addition to calculating molar percentages from $\mathrm{CH} / \mathrm{CDs}$, we can also compute absolute concentrations with aggregate intensities in the $\mathrm{C}-\mathrm{D}$ channel and our reference calibration curves (Figure 1d). Such quantification is otherwise highly challenging for existing methods. We selected three representative aggregates with different sizes for calculation (Figure 4a, color-indicated, details in the SI and results listed in Table S1). The mHtt protein concentration in the small aggregate (Figure 4a, green dot) ranges from 1.2 to $2.1 \mathrm{mM}$ (Figure 4c, $6.7 \mu \mathrm{m}^{2}$ ). It increases to $4.3-5.1 \mathrm{mM}$ (and Figure $4 \mathrm{~d}, 19.1$ $\mu \mathrm{m}^{2}$ ) for the medium aggregate (Figure $4 \mathrm{a}$, red dot). The concentration becomes 5.6-6.4 $\mathrm{mM}$ for the large aggregate (36.5 $\mu \mathrm{m}^{2}$, Figure 4a, magenta dot). The upper and lower limits shown are determined by the relative content of newly synthesized and pre-existing non-mHtt proteins sequestered to the aggregates. Surprisingly, we found that while $\mathrm{mHtt}$ concentrations increase with aggregate sizes, the concentrations of non-mHtt proteins remain almost the same for small $(4.6 \mathrm{mM})$, medium $(4.7 \mathrm{mM})$, and large $(4.2 \mathrm{mM})$ aggregates (Table S1). Our observations therefore suggest that the formation of small aggregates preferentially sequesters non-

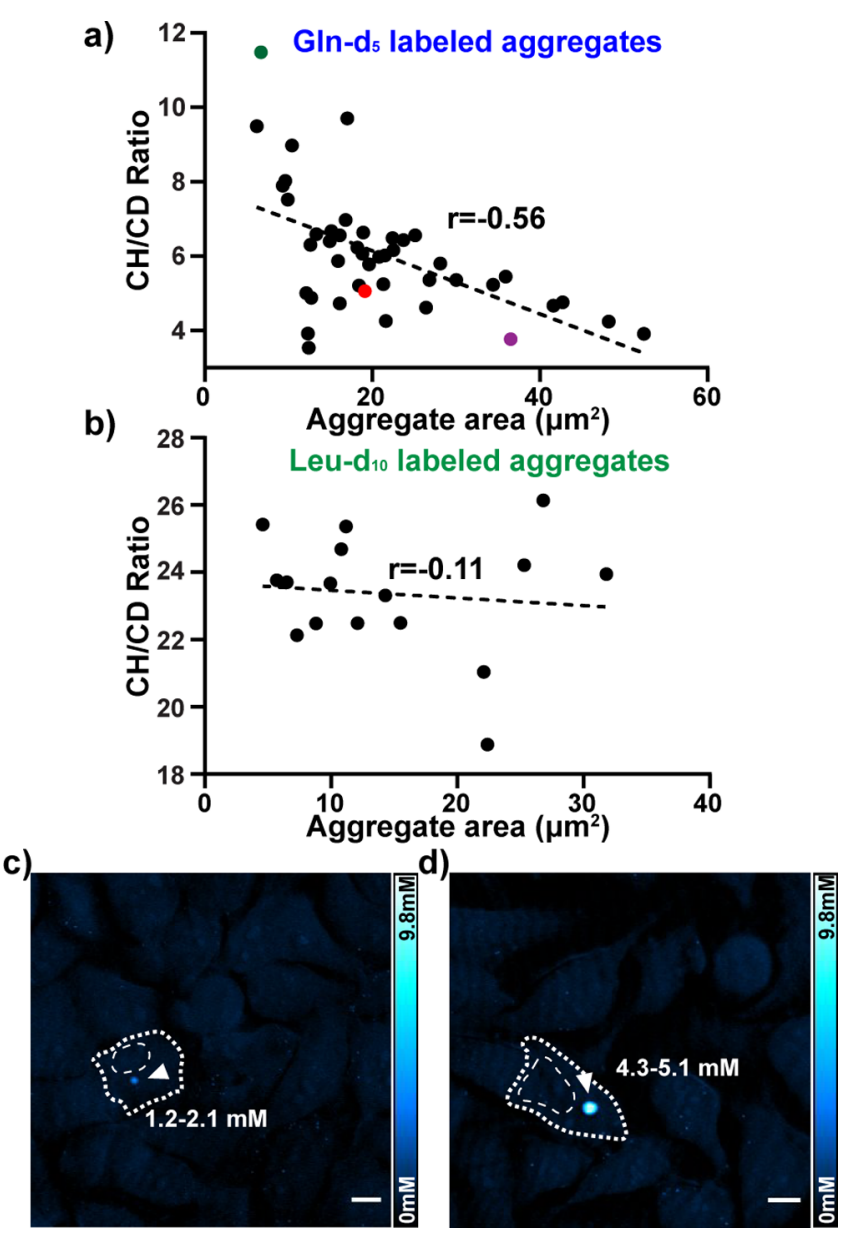

Figure 4. SRS quantification for mHtt-97Q aggregates of different sizes. (a) $\mathrm{CH} / \mathrm{CDs}$ for $\mathrm{Gln}-d_{5}$-labeled aggregates present a negative correlation on aggregation areas (Pearson's coefficient $r=-0.56$ ). (b) Minimum correlation between $\mathrm{CH} / \mathrm{CDs}$ for Leu- $d_{10}$-labeled aggregates and aggregation areas (Pearson's coefficient $r=-0.11$ ). (c, d) C-D SRS images for representative Gln- $d_{5}$-labeled small $\left(c, 6.7 \mu \mathrm{m}^{2}\right.$, green dot in a) and medium (d, $19.1 \mu \mathrm{m}^{2}$, red dot in a) mHtt-97Q aggregates. Calculated $\mathrm{mHtt}-97 \mathrm{Q}$ concentrations for the aggregates are indicated. Cell shapes and nuclei are outlined by a white dotted line. Scale bar: $10 \mu \mathrm{m}$.

mHtt cytosolic proteins (to as high as $70 \%$ ). These proteins are likely functional chaperones, ribonucleoproteins, and structural proteins. ${ }^{9-13}$ As the aggregates become larger, they then sequester more $\mathrm{mHtt}$ proteins. This might indicate a cellular rescue mechanism during aggregate growth by clearing toxic mHtt proteins. ${ }^{4,7,8}$ Our data further demonstrate that the total protein concentrations of these aggregates fall in the range $5-10 \mathrm{mM}$. To the best of our knowledge, our study is the first to quantify the mHtt protein concentrations and molar percentages for aggregates of different sizes in live cells.

Interrogating Aggregate Structures and AggregateEnvironment Interactions by Hyperspectral SRS (hSRS) Imaging. One key advantage for in vitro spectroscopic studies over live-cell fluorescence imaging is that they provide structural and conformational information on the aggregates. $^{14-19}$ For instance, multiple reports suggested the presence of $\beta$-sheet-rich structures in $\mathrm{mHtt}$ aggregates. However, there is still no consensus. ${ }^{15,18}$ We therefore adopted hSRS to analyze mHtt-97Q aggregates in live cells. We started with the amide I band since its Raman vibration is well 

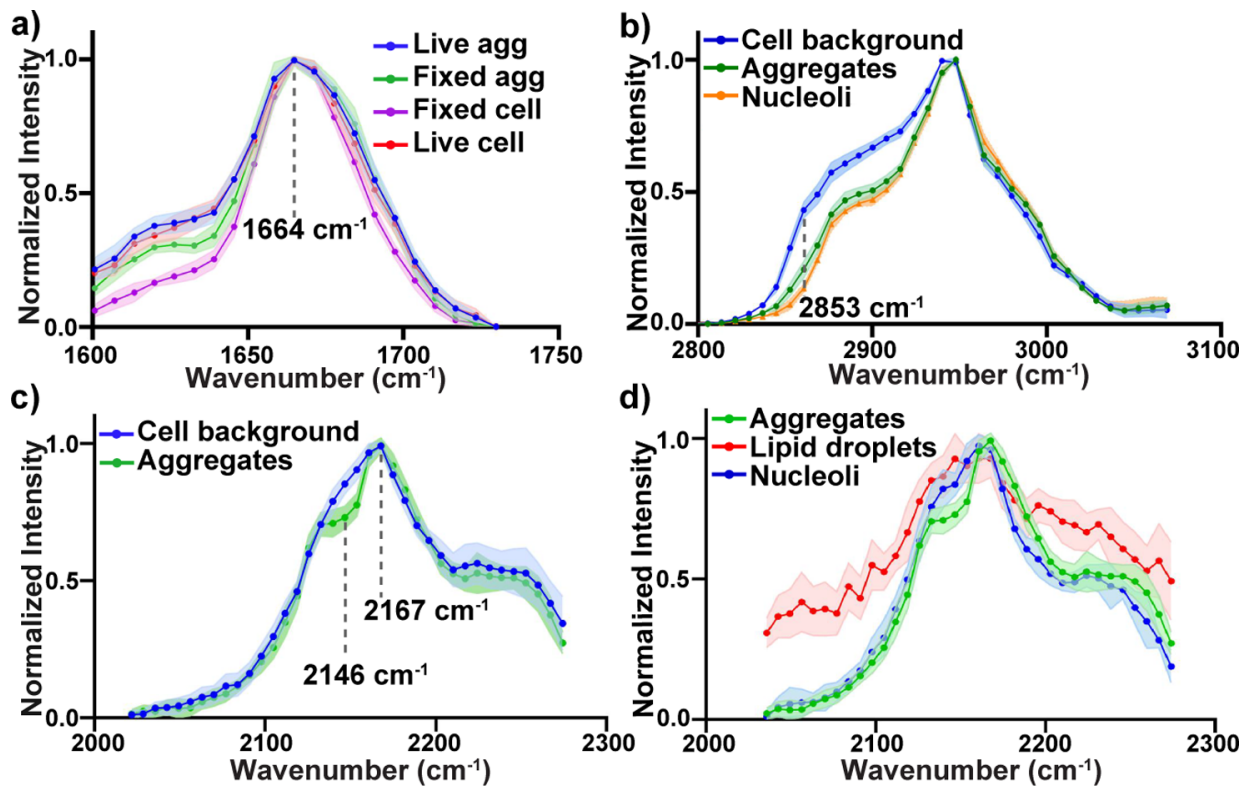

Figure 5. Hyperspectral SRS analysis on mHtt-97Q aggregates. (a) Average amide I SRS spectra of live aggregates (Live agg, blue, $n=14$ ), live cellbackground (Live cell, red, $n=14$ ), fixed aggregates (Fixed agg, green, $n=10$ ), and fixed cell-background (Fixed cell, magenta, $n=10$ ). (b) Livecell SRS $\mathrm{CH}_{3}$ spectra of aggregates (green, $n=14$ ), cell background (blue, $n=16$ ), and nucleoli (orange, $n=16$ ). (c) Live-cell SRS C-D spectra of Gln- $d_{5}$-labeled aggregates (green, $n=20$ ) and cell background (blue, $\left.n=20\right)$. (d) Live-cell SRS C $-\mathrm{D}$ spectra on small mHtt aggregates $($ green, $n=$ 20), lipid droplets (red, $n=10)$, and nucleoli (blue, $n=12$ ) with distinct spectral features. Error bar: SD.
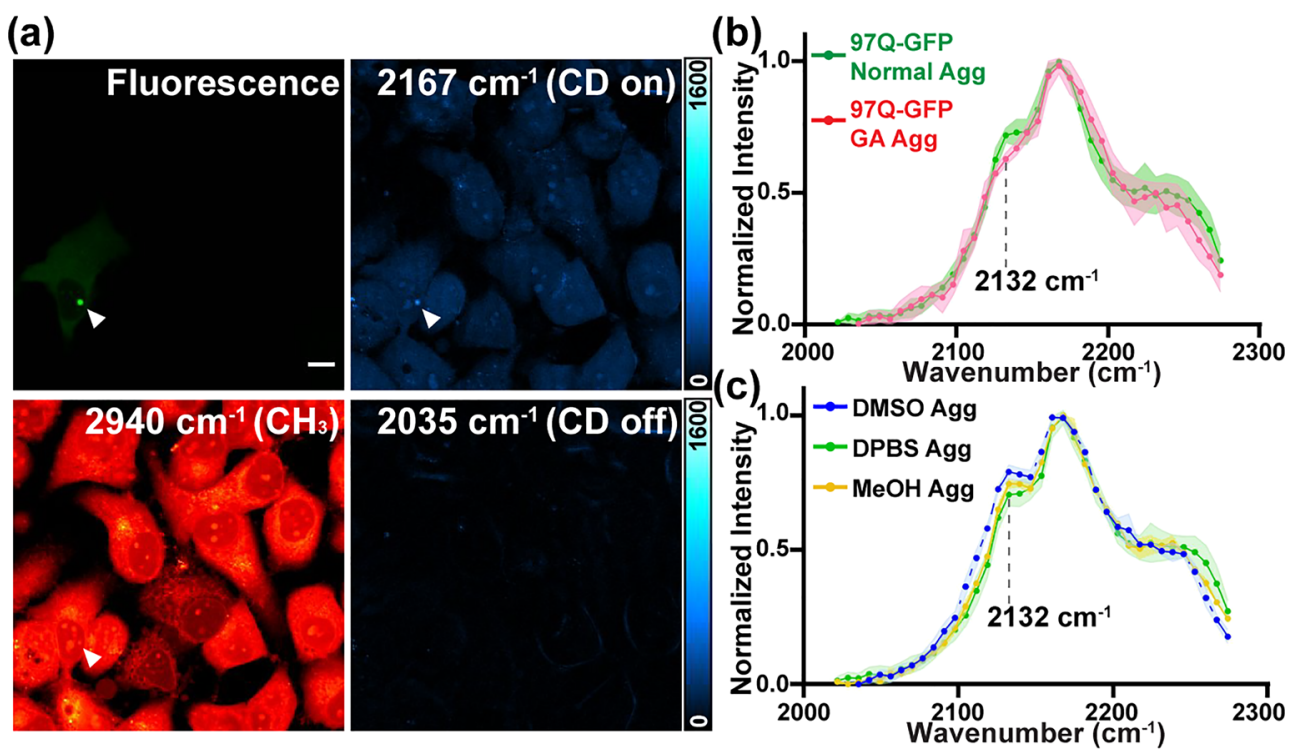

Figure 6. Hyperspectral SRS study of mHtt aggregations upon cellular heat shock responses. (a) Fluorescence (green) and correlative SRS images for mHtt-97Q-GFP aggregates (white arrowheaded) at $2167 \mathrm{~cm}^{-1}$ (C-D on-resonance), $2035 \mathrm{~cm}^{-1}$ (off-resonance), and $2940 \mathrm{~cm}^{-1}\left(\mathrm{CH}_{3}\right)$ in live cells after $100 \mathrm{nM}$ geldanamycin (GA) treatment for $20 \mathrm{~h}$. Scale bar: $10 \mu \mathrm{m}$. (b) SRS spectra of normal mHtt aggregates (green, $n=20)$ and a subset of GA-treated small aggregates (red, $n=7)$. (c) SRS spectra of $\mathrm{mHtt}$ aggregates in fixed cells in DPBS buffer $($ green, $n=15), \mathrm{MeOH}$ (yellow, $n=5$ ), and DMSO (blue, $n=6$ ). Error bar: SD.

documented for probing protein secondary structures by vibrational spectroscopy. ${ }^{16}$ A recent report by label-free hSRS imaging of amide I on amyloid plaques in brain tissues revealed a clear $12 \mathrm{~cm}^{-1}$ blue shift (resolvable by our spectral resolution of $\sim 12 \mathrm{~cm}^{-1}$ ), which corresponds to cross- $\beta$ sheet structures. ${ }^{35}$ Nonetheless, we observed no such difference comparing the amide I spectrum of mHtt aggregates to that of the cellular background (Figure 5a, $1664 \mathrm{~cm}^{-1}$, blue vs red). Similar to a recent study, ${ }^{18}$ our data suggest that $\beta$-sheet structures might not be enriched in these aggregates. Note that we detected a spectral distortion upon chemical fixation (Figure 5a, from 1600 to $1640 \mathrm{~cm}^{-1}$, green vs blue and magenta vs red), highlighting the importance of live-sample analysis. ${ }^{35,36}$ Similar to amide $\mathrm{I}, \mathrm{CH}_{3}\left(2940 \mathrm{~cm}^{-1}\right)$ offers no discernible spectral information (Figure $5 b$ ), since the signals mainly come as sequestered non-mHtt proteins. The aggregates' spectra are almost identical to those of nucleoli with abundant proteins and rRNA. The lower shoulder at $2850 \mathrm{~cm}^{-1}$, attributed to $\mathrm{CH}_{2}$ vibrations, implies reduced lipids in the aggregates. 
We next asked whether the $\mathrm{C}-\mathrm{D}$ spectral region could offer insightful structural information with specific aggregate labeling. Interestingly, we observed a clear dip around $2146 \mathrm{~cm}^{-1}$ from aggregates compared to that from the cellular background (Figure 5c), suggesting a structural or microenvironmental difference between aggregated and cytosolic $\mathrm{mHtt}$ proteins. To understand such spectral changes, we fitted the averaged spectra (Figure 5c) by three Lorentzian peaks (Figure S11a,b). Our results indicate that the observed $2146 \mathrm{~cm}^{-1}$ dip resulted from the narrowing and the slight red shift of the shoulder peak at $2130 \mathrm{~cm}^{-1}$ (Figure S11c,d). The narrowing of the peak width is likely due to the formation of a more ordered structure upon aggregation. ${ }^{16}$ Our results establish that this shoulder peak could serve as a sensitive indicator for the microenvironment of mHtt aggregates. We note that the weak-intensity cellular background structures, including nucleoli and lipid droplets shown in the $\mathrm{C}-\mathrm{D}$ images, might interfere with the specific analysis of small aggregates with low signals. In this case, C-D spectra generated by hSRS on aggregates, nucleoli, and lipid droplets could help unequivocally differentiate these structures (Figure 5d).

As we have proven our technique as an effective method to analyze the microenvironment of native aggregates, we sought to apply it to understanding molecular interactions between polyQ aggregates and heat shock proteins (HSPs), key chaperones for protein folding and inhibiting protein aggregations. $^{37}$ This may offer fundamental insight toward therapeutic development for HD. ${ }^{6}$ We induced cellular heat shock responses by a small-molecule drug, geldanamycin (GA), an inhibitor of HSP 90, which has been shown to effectively induce the expression of HSP 40 and 70 for the clearance of polyQ aggregates. ${ }^{37}$ We first validated the function of GA by confirming that the number of large aggregates decreases with the treatment of increased GA concentrations ${ }^{37}$ (Figure S12). We next resorted to C-D hSRS on mHtt-97Q aggregates. Interestingly, in GA-treated samples, we discovered a subset of aggregates with reduced size and intensity that consistently present a varied spectral feature with a lowered shoulder peak at $2132 \mathrm{~cm}^{-1}$ compared to that of larger-size aggregates (Figure S13a,b). Inspired by this spectroscopic observation, we came back to mHtt-97Q-GFP and adopted a correlative fluorescence and hSRS to understand this phenomenon (Figure 6a). We found that such aggregates, when exhibiting the same SRS spectral feature as found for the mHtt-97Q subset (Figure 6b and Figure S13c), always coexist with surrounding diffusive $\mathrm{mHtt}$ protein pools (Figure 6a, Fluorescence). Recently, a liquid-to-solid phase transition is reported for the mHtt-97Q-GFP proteins during aggregation formation. ${ }^{38}$ We first speculated that our observed shoulder peak decrease is caused by a reverse phase conversion from solid $\mathrm{mHtt}$ aggregates toward the surrounding liquid pool by GA triggered upregulation of HSP 40/70. However, the fluorescence loss in photobleaching (FLIP) experiments on the surrounding fluorescent pool did not reveal any fluorescence exchange between the cytosolic and aggregated $\mathrm{mHtt}$ proteins (Figure S14), ruling out our phase-transition hypothesis.

Since $\mathrm{C}-\mathrm{D}$ vibration is sensitive to the microenvironment, similar to that from $\mathrm{CH}^{39}$ we then asked whether such a decrease at the $2132 \mathrm{~cm}^{-1}$ shoulder peak corresponds to a change of secondary structures when interacting with HSP 40/ 70 proteins, as suggested by previous in vitro EM experiments. ${ }^{40}$ To test the hypothesis, we emerged fixed non-GAtreated cells containing mHtt-97Q-GFP aggregates in solvents with descending hydrogen-bonding capacity (Figure 6c, from DPBS buffer (green), to $\mathrm{MeOH}$ (yellow) and DMSO (blue)). Interestingly, the $2132 \mathrm{~cm}^{-1}$ shoulder increases accordingly (Figure 6c, vertical line). Quantitative fittings for the averaged spectra with three Lorentzian peaks (Figure S15a-c) further confirmed an increase of ratios between the shoulder (2130 $\mathrm{cm}^{-1}$, peak1) and the major $\left(2169 \mathrm{~cm}^{-1}\right.$, peak2) peaks (Figure $\mathrm{S} 15 \mathrm{e}, \mathrm{f})$ from DPBS-treated aggregates (Figure S15a) to DMSO-treated aggregates (Figure S15c). This indicates that the peak1/peak2 ratios may report the local solvation states (e.g., hydrogen bonding) of aggregates. Similar fitting for the averaged spectrum from GA-treated aggregates (Figure S15d) showed that it poses the lowest peak1/peak2 ratio (Figure $\mathrm{S} 15 \mathrm{e}, \mathrm{f})$, suggesting that the local solvation environment for our captured GA-treated aggregates may be more hydrogenbonded compared to that for regular cellular proteomes (Figure S15d). Such likely hyperhydration status might be caused by a partially folded state, a folding intermediate, for aggregates upon interacting with HSP 40/70 in live cells. ${ }^{41}$ We further analyzed the $\mathrm{CH} / \mathrm{CD}$ ratios on these small aggregates in cells with and without induced heat-shock responses. We postulated that if the heat-shock responses recruit extra HSPs to the aggregates, as our hSRS data suggested above, we might detect an increase in $\mathrm{CH} / \mathrm{CD}$ ratios since the percentage of the non-mHtt proteins is higher in these aggregates. We indeed found that the $\mathrm{CH} / \mathrm{CD}$ ratios became slightly higher in small aggregates from cells under induced heat-shock responses compared to those in similarly sized aggregates from noninduced cells (Figure S16).

\section{- CONCLUSIONS}

In summary, we demonstrated the combination of SRS microscopy with Gln- $d_{5}$ labeling to be a general platform for sensitive and specific imaging, ratiometric quantification, and hyperspectral analysis of native polyQ aggregates in HD. Our technique provides new and complementary structural and compositional information to our current knowledge of polyQ aggregates. As shown above, our detection sensitivity and specificity are ensured from three aspects. First, the expanded polyQ sequences yield a Gln- $d_{5}$ labeling enrichment in disease proteins over the normal proteins with only low $\mathrm{Q}$ abundance. Second, the formation of the aggregates is specifically induced by these disease-form polyQ proteins. Together, these ensure a much higher signal from polyQ aggregates compared to that from the cellular background. Third, in the case the aggregates are small with low signals, hSRS on aggregates adds an additional layer of spectral specificity, differentiating the aggregates from other puncta-like structures, including nucleoli and lipid droplets.

Our method is also applicable to polyQ expansions of various lengths. In particular, with linear concentration dependence, it is suited for investigating aggregates of extended polyQ construct (e.g., >200Q), which may form rather dense structures and pose challenges to study by other strategies. Our method is also applicable to other polyQ diseases, including spinocerebellar ataxia and spinobulbar muscular atrophy. ${ }^{42}$ Other poly aa diseases ${ }^{43,44}$ with polyglycine-alanine (poly-GA), poly-proline-arginine (poly-PR), and poly-proline-alanine (poly-PA) aggregates, recently reported in the ALS/FTD patient brain, could also be investigated by selective deuteration of corresponding aa. To better guide future SRS work on these aggregates, we further calculated the corresponding SRS detection limits for 
representative, varying lengths of polyQ and poly aa sequences (Table S2). In addition, since Gln can transport across the blood-brain barrier, ${ }^{45}$ and deuterium labeling is minimally invasive, applications to animal models or even to humans may be possible. Moreover, correlative live-cell SRS imaging with Gln- $d_{5}$ labeling with recently demonstrated Cryo-ET ${ }^{11}$ or quantitative proteomics ${ }^{12}$ may offer a comprehensive structure-function relationship for native $\mathrm{mHtt}-97 \mathrm{Q}$ aggregates.

\section{ASSOCIATED CONTENT}

\section{SI Supporting Information}

The Supporting Information is available free of charge at https://pubs.acs.org/doi/10.1021/acscentsci.9b01196.

Detailed experimental procedures, calculations for detection limits and compositional analysis, supplementary tables, and supplementary figures (PDF)

\section{AUTHOR INFORMATION}

\section{Corresponding Author}

Lu Wei - Division of Chemistry and Chemical Engineering, California Institute of Technology, Pasadena, California 91125,

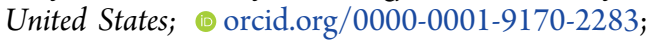
Email: lwei@caltech.edu

\section{Author \\ Kun Miao - Division of Chemistry and Chemical Engineering, California Institute of Technology, Pasadena, California 91125, United States; ○ orcid.org/0000-0001-6567-3650}

Complete contact information is available at:

https://pubs.acs.org/10.1021/acscentsci.9b01196

\section{Notes}

The authors declare no competing financial interest.

\section{ACKNOWLEDGMENTS}

We would like to thank Dr. C. Qian, D. Lee, J. Du, and Dr. L. Voong for helpful discussions. We are grateful for the plasmid (mHtt-97Q-GFP) shared by Prof. R. Kopito and Prof. F.-U. Hartl. We thank Prof. Z. Liu for sharing the stable embryonic stem cell-lines. L.W. acknowledges the support of start-up funds from California Institute of Technology.

\section{REFERENCES}

(1) Paulson, H. Protein fate in neurodegenerative proteinopathies: polyglutamine diseases join the (mis)fold. Am. J. Hum. Genet. 1999, 64, 339-345.

(2) Hipp, M. S.; Park, S.-H.; Hartl, F. U. Proteostasis impairment in protein-misfolding and -aggregation diseases. Trends Cell Biol. 2014, 24 (9), 506-514.

(3) Bates, G. Huntingtin 1aggregation and toxicity in Huntington's disease. Lancet 2003, 361 (9369), 1642-1644.

(4) Finkbeiner, S. Huntington's disease. Cold Spring Harbor Perspect. Biol. 2011, 3, No. a007476.

(5) Sahl, S. J.; Lau, L.; Vonk, W. I. M.; Weiss, L. E.; Frydman, J.; Moerner, W. E. Delayed emergence of subdiffraction-sized mutant Huntingtin fibrils following inclusion body formation. Q. Rev. Biophys. 2016, 49 (e2), 1-13.

(6) Eisele, Y. S.; Monteiro, C.; Fearns, C.; Encalada, S. E.; Wiseman, R. L.; Powers, E. T.; Kelly, J. W. Targeting protein aggregation for the treatment of degenerative diseases. Nat. Rev. Drug Discovery 2015, 14 (11), 759-780.

(7) Ramdzan, Y. M.; Trubetskov, M. M.; Ormsby, A. R.; Newcombe, E. A.; Sui, X.; Tobin, M. J.; Bongiovanni, M. N.; Gras, S. L.; Dewson, G.; Miller, J. M. L.; et al. Huntingtin inclusions trigger cellular quiescence, deactivate apoptosis, and lead to delayed necrosis. Cell Rep. 2017, 19 (5), 919-927.

(8) Arrasate, M.; Mitra, S.; Schweitzer, E. S.; Segal, M. R.; Finkbeiner, S. Inclusion Body Formation Reduces Levels of Mutant Huntingtin and the Risk of Neuronal Death. Nature 2004, 431 (7010), 805-810.

(9) Hosp, F.; Gutiérrez-Ángel, S.; Schaefer, M. H.; Cox, J.; Meissner, F.; Hipp, M. S.; Hartl, F.-U.; Klein, R.; Dudanova, I.; Mann, M. Spatiotemporal proteomic profiling of Huntington's disease inclusions reveals widespread loss of protein function. Cell Rep. 2017, 21 (8), 2291-2303.

(10) Suhr, S. T.; Senut, M.-C.; Whitelegge, J. P.; Faull, K. F.; Cuizon, D. B.; Gage, F. H. Identities of sequestered proteins in aggregates from cells with induced polyglutamine expression. J. Cell Biol. 2001, 153 (2), 283-294.

(11) Bäuerlein, F. J. B.; Saha, I.; Mishra, A.; Kalemanov, M.; Martínez-Sánchez, A.; Klein, R.; Dudanova, I.; Hipp, M. S.; Hartl, F. U.; Baumeister, W.; et al. In situ architecture and cellular interactions of polyQ inclusions. Cell 2017, 171, 179-187.

(12) Olzscha, H.; Schermann, S. M.; Woerner, A. C.; Pinkert, S.; Hecht, M. H.; Tartaglia, G. G.; Vendruscolo, M.; Hayer-Hartl, M.; Hartl, F. U.; Vabulas, R. M. Amyloid-like aggregates sequester numerous metastable proteins with essential cellular functions. Cell 2011, 144, 67-78.

(13) Donaldson, K. M.; Li, W.; Ching, K. A.; Batalov, S.; Tsai, C.-C.; Joazeiro, C. A. P. Ubiquitin-mediated sequestration of normal cellular proteins into polyglutamine aggregates. Proc. Natl. Acad. Sci. U. S. A. 2003, 100 (15), 8892-8897.

(14) Buchanan, L. E.; Carr, J. K.; Fluitt, A. M.; Hoganson, A. J.; Moran, S. D.; de Pablo, J. J.; Skinner, J. L.; Zanni, M. T. Structural motif of polyglutamine amyloid fibrils discerned with mixed-isotope infrared spectroscopy. Proc. Natl. Acad. Sci. U. S. A. 2014, 111 (16), $5796-5801$.

(15) Xiong, K.; Punihaole, D.; Asher, S. A. UV resonance Raman spectroscopy monitors polyglutamine backbone and side chain hydrogen bonding and fibrillization. Biochemistry 2012, 51, 58225830 .

(16) Devitt, G.; Howard, K.; Mudher, A.; Mahajan, S. Raman Spectroscopy: An emerging tool in neurodegenerative disease research and diagnosis. ACS Chem. Neurosci. 2018, 9 (3), 404-420.

(17) Matlahov, I.; van der Wel, P. C. Conformational Studies of Pathogenic Expanded Polyglutamine Protein Deposits from Huntington's Disease. Exp. Biol. Med. 2019, 244, 1584.

(18) Warner, J. B.; Ruff, K. M.; Tan, P. S.; Lemke, E. A.; Pappu, R. V.; Lashuel, H. A. Monomeric Huntingtin exon 1 has similar overall structural features for wild-type and pathological polyglutamine lengths. J. Am. Chem. Soc. 2017, 139 (41), 14456-14469.

(19) Crick, S. L.; Jayaraman, M.; Frieden, C.; Wetzel, R.; Pappu, R. $\mathrm{V}$. Fluorescence correlation spectroscopy shows that monomeric polyglutamine molecules form collapsed structures in aqueous solutions. Proc. Natl. Acad. Sci. U. S. A. 2006, 103 (45), 16764-16769.

(20) Iwata, A.; Christianson, J. C.; Bucci, M.; Ellerby, L. M.; Nukina, N.; Forno, L. S.; Kopito, R. R. Increased susceptibility of cytoplasmic over nuclear polyglutamine aggregates to autophagic degradation. Proc. Natl. Acad. Sci. U. S. A. 2005, 102 (37), 13135-13140.

(21) Liu, Y.; Wolstenholme, C. H.; Carter, G. C.; Liu, H.; Hu, H.; Grainger, L. S.; Miao, K.; Fares, M.; Hoelzel, C. A.; Yennawar, H. P.; et al. Modulation of fluorescent protein chromophores to detect protein aggregation with turn-on fluorescence. J. Am. Chem. Soc. 2018, 140, 7381-7384.

(22) Mangiarini, L.; Sathasivam, K.; Seller, M.; Cozens, B.; Harper, A.; Hetherington, C.; Lawton, M.; Trottier, Y.; Lehrach, H.; Davies, S. W.; et al. Exon 1 of the HD gene with an expanded CAG repeat is sufficient to cause a progressive neurological phenotype in transgenic mice. Cell 1996, 87, 493-506.

(23) Tsien, R. Y. The Green Fluorescent Protein. Annu. Rev. Biochem. 1998, 67 (1), 509-544. 
(24) Bhattacharya, M.; Mukhopadhyay, S. Studying Protein Misfolding and Aggregation by Fluorescence Spectroscopy. In Reviews in Fluorescence 2015; Geddes, C., Ed.; Springer: Cham, 2016; Vol. 8.

(25) Kozlowski, L. P. Proteome-pI: proteome isoelectric point database. Nucleic Acids Res. 2017, 45, D1112-D1116.

(26) Freudiger, C. W.; Min, W.; Saar, B. G.; Lu, S.; Holtom, G. R.; He, C.; Tsai, J. C.; Kang, J. X.; Xie, X. S. Label-free biomedical imaging with high sensitivity by stimulated Raman scattering microscopy. Science 2008, 322 (5909), 1857-1861.

(27) Shen, Y.; Xu, F.; Wei, L.; Hu, F.; Min, W. Live-cell quantitative imaging of proteome degradation by stimulated Raman scattering. Angew. Chem., Int. Ed. 2014, 53, 5596-5599.

(28) Wei, L.; Shen, Y.; Xu, F.; Hu, F.; Harrington, J. K.; Targoff, K. L.; Min, W. Imaging complex protein metabolism in live organisms by stimulated Raman scattering microscopy with isotope labeling. ACS Chem. Biol. 2015, 10 (3), 901-908.

(29) Zhang, J.; Yan, S.; He, Z.; Ding, C.; Zhai, T.; Chen, Y.; Li, H.; Yang, G.; Zhou, X.; Wang, P. Small unnatural amino acid carried Raman tag for molecular imaging of genetically targeted proteins. $J$. Phys. Chem. Lett. 2018, 9 (16), 4679-4685.

(30) Min, W.; Freudiger, C. W.; Lu, S.; Xie, X. S. Coherent Nonlinear Optical Imaging: Beyond Fluorescence Microscopy. Annu. Rev. Phys. Chem. 2011, 62 (1), 507-530.

(31) Perney, N. M.; Braddick, L.; Jurna, M.; Garbacik, E. T.; Offerhaus, H. L.; Serpell, L. C.; Blanch, E.; Holden-Dye, L.; Brocklesby, W. S.; Melvin, T. Polyglutamine aggregate structure in vitro and in vivo; new avenues for coherent anti-stokes Raman scattering microscopy. PLoS One 2012, 7 (7), No. e40536.

(32) Wei, L.; Hu, F.; Shen, Y.; Chen, Z.; Yu, Y.; Lin, C.-C.; Wang, M. C.; Min, W. Live-cell imaging of alkyne-tagged small biomolecules by stimulated Raman scattering. Nat. Methods 2014, 11 (4), 410-412.

(33) Piez, K. A.; Eagle, H. The free amino acid pool of cultured human cells. J. Bol. Chem. 1958, 231, 533-545.

(34) Li, L.; Liu, H.; Dong, P.; Li, D.; Legant, W. R.; Grimm, J. B.; Lavis, L. D.; Betzig, E.; Tjian, R.; Liu, Z. Real-time imaging of Huntingtin aggregates diverting target search and gene transcription. eLife 2016, 5, 17056.

(35) Ji, M.; Arbel, M.; Zhang, L.; Freudiger, C. W.; Hou, S. S.; Lin, D.; Yang, X.; Bacskai, B. J.; Xie, X. S. Label-free imaging of amyloid plaques in Alzheimer's disease with stimulated Raman scattering microscopy. Sci. Adv. 2018, 4, No. eaat7715.

(36) Hackett, M. J.; McQuillan, J. A.; El-Assaad, F.; Aitken, J. B.; Levina, A.; Cohen, D. D.; Siegele, R.; Carter, E. A.; Grau, G. E.; Hunt, N. H.; et al. Chemical alterations to murine brain tissue induced by formalin fixation: implications for biospectroscopic imaging and mapping studies of disease pathogenesis. Analyst 2011, 136, 29412952.

(37) Sittler, A.; Lurz, R.; Lueder, G.; Priller, J.; Hayer-Hartl, M. K.; Hartl, F. U.; Lehrach, H.; Wanker, E. E. Geldanamycin activates a heat shock response and inhibits Huntingtin aggregation in a cell culture model of Huntington's disease. Hum. Mol. Genet. 2001, 10 (12), 1307-1315.

(38) Peskett, T. R.; Rau, F.; O’Driscoll, J.; Patani, R.; Lowe, A. R.; Saibil, H. R. A liquid to solid phase transition underlying pathological Huntingtin exon1 aggregation. Mol. Cell 2018, 70, 588-601.

(39) Lee, H. J.; Zhang, D.; Jiang, Y.; Wu, X.; Shih, P.-Y.; Liao, C.-S.; Bungart, B.; Xu, X.-M.; Drenan, R.; Bartlett, E.; et al. Label-free vibrational spectroscopic imaging of neuronal membrane potential. J. Phys. Chem. Lett. 2017, 8, 1932-1936.

(40) Muchowski, P. J.; Schaffar, G.; Sittler, A.; Wanker, E. E.; HayerHartl, M. K.; Hartl, F. U. Hsp70 and Hsp40 chaperones can inhibit self-assembly of polyglutamine proteins into amyloid-like fibrils. Proc. Natl. Acad. Sci. U. S. A. 2000, 97 (14), 7841-7846.

(41) Hartl, F. U.; Bracher, A.; Hayer-Hartl, M. Molecular chaperones in protein folding and proteostasis. Nature 2011, 475, 324-332.

(42) Orr, H. T.; Zoghbi, H. Y. Trinucleotide repeat disorders. Annu. Rev. Neurosci. 2007, 30, 575-621.
(43) Guo, Q.; Lehmer, C.; Martínez-Sánchez, A.; Rudack, T.; Beck, F.; Hartmann, H.; Pérez-Berlanga, M.; Frottin, F.; Hipp, M. S.; Hartl, F. U.; et al. In situ structure of neuronal C9orf72 poly-GA aggregates reveals proteasome recruitment. Cell 2018, 172, 696-705.

(44) Ash, P. E. A.; Bieniek, K. F.; Gendron, T. F.; Caulfield, T.; Lin, W.-L.; DeJesus-Hernandez, M.; van Blitterswijk, M. M.; Jansen-West, K.; Paul, J. W.; Rademakers, R.; et al. Unconventional translation of C9orf72 GGGGCC expansion generates insoluble polypeptides specific to c9FTD/ALS. Neuron 2013, 77, 639-646.

(45) Ennis, S. R.; Kawai, N.; Ren, X.; Abdelkarim, G. E.; Keep, R. F. Glutamine Uptake at the Blood-Brain Barrier Is Mediated by NSystem Transport. J. Neurochem. 1998, 71 (6), 2565-2573. 